\title{
Cytology-histology correlation of gynecologic and non-gynecologic samples at a tertiary health care center: A two-year study
}

\author{
Kalyani Raju*, Supreetha Megalamane Sunanda, Varsha Shree Rajanna
}

Department of Pathology, Sri Devaraj Urs Medical College, Sri DevarajUrs Academy of Higher Education and Research, Kolar, Karnataka, India

\section{Correspondence}

Kalyani Raju, Department of Pathology, Sri Devaraj Urs Medical College, Sri DevarajUrs Academy of Higher Education and Research, Kolar, Karnataka, India

Email: rkalyanir@rediffmail.com History

- Received: 02 February 2019

- Accepted: 25 March 2019

- Published: 29 April 2019

DOI :

https://doi.org/10.15419/bmrat.v6i4.534

\section{Check for updates}

\section{Copyright}

(c) Biomedpress. This is an openaccess article distributed under the terms of the Creative Commons Attribution 4.0 International license.

\section{BioMedPress} The Open Access Publisher

\begin{abstract}
Background: Cytology-histology correlation is a method of detection of an error in the diagnostic field. The root cause analysis is done for the discordant cases for quality improvement of the laboratory reports. Aims: To find out the root cause in discordant cytology-histology case pairs. Methods: This is a Retrospective cross-sectional descriptive study. The study was done from January 2016 to December 2017. All FNAC and Pap test cases which had corresponding biopsy slides and reports are included in the study. The discordant cases were classified as sampling error, interpretation error, cytology error, and histopathology error. Descriptive statistical analysis was used for the analysis of results. Results: Of total 639 case pairs, gynecologic case pairs were 447, and nongynecologic case pairs were 192. Among the gynecologic cases, the concordance between cytology and histopathology diagnosis were 425 (95\%) and discordance 22 (5\%) of which discrepancy in cytology was 11, histopathology was seven, and both cytology and histopathology error was four. The interpretation error was 14, and the sampling error was nine. Among non-gynecologic cases, the concordance between cytology and histopathology diagnosis were 167 (86\%) and discordance 25 (14\%) of which discrepancy in cytology was 17, histopathology was seven, and both cytology and histopathology error was one. The interpretation error was 16, and the sampling error was ten. The discordance versus concordance ratio was 1:19.3, 1:6.68 and 1:12.5 for gynecology, non-gynecology, and total case pairs respectively. Conclusions: The percentage of error in gynecologic cases was within the range of published data. However, those of non-gynecology cases were slightly higher. Regular professional training and improvement of sampling techniques especially the guided FNAC can reduce the errors.
\end{abstract}

Key words: Cytology, Cytology-Histology correlation, Quality assurance in cytology

\section{INTRODUCTION}

Cytology-histology correlation (CHC) is a method of detection of an error in the diagnostic field where the cytology interpretations are compared with the histopathology interpretation of the specimens from the same site. It assesses the concordance and discordance of the interpretation, regulated by CLIA 1998 as laboratory quality control (QC) programme dealing with examination and reporting the results in cytology laboratory. It enhances the accuracy and precision of cytology reports. It is a system based procedure usually a retrospective activity done in a passive manner. CHC detects and determines the frequency of error, type of error and also the severity of clinical outcome associated with the error ${ }^{1-3}$.

The root cause analysis (RCA) is done for the discordant cases to detect the cause of an error. The causes for the discordance can be biologic variation, issues of sampling quality, processing of samples and challenges in interpretative ${ }^{3,4}$. The error depends on some factors as; institution-specific factors, patient factors, look back time period, type of biopsy, reproducible/non-reproducible reports, etc. ${ }^{1-3}$.

$\mathrm{CHC}$ has evolved in the past decade and is highly valuable in fields of cytology and histopathology to improve diagnostic testing and screening process. It is one of the quality indicators of the cytology lab ${ }^{4,5}$. Hence, we have taken up this study. The objectives of the study are; to estimate the percentage of concordance and discordance of cytology and histopathology reports in gynecology and non-gynecology samples and to classify the cause for discordance as sampling error or interpretation error.

\section{METHODS}

This study was a retrospective cross-sectional descriptive study conducted from 5906 January 2016 to December 2017 at Central Diagnostic Laboratory attached to a teaching hospital in the southern part of Karnataka. The institutional ethical clearance has 
been taken before the start of the study. The inclusion criteria were all fine needle aspiration cytology (FNAC) and Pap test cases which had the corresponding biopsy, both cytology and histopathology having slides and reports which were from the same anatomic sites. The exclusion criteria were cases with pre-analytical errors and incomplete test request forms where the patient details were not clear.

The cytology and biopsy slides were retrieved from the archives of the Pathology department and screened by two Pathologists. The Pap smears were categorized by semi-quantitative measures shown in Table 1 . The FNAC reports were categorized by semi-quantitative measure as shown in Table 2. Both the tables are modified version of that used by Raab et al. ${ }^{1}$. In case of disparity of opinion between Pathologists, the slides were screened again and the consensus has arrived. The cases were analyzed for the percentage of concordance and discordance separately for gynecologic and non-gynecologic cases.

RCA was for discordant cases were done by dichotomous assessment as sampling error and interpretation error. If the diagnostic material was not available or is non-representative in biopsy slides and the interpretation is appropriate, then it was taken as sampling error. If the diagnostic equipment is available and representative in the biopsy slides and is not interpreted appropriately, then it was taken as an interpretation error. The discordant cases were assessed site/organwise, the time interval between cytology report and biopsy sample, change of diagnosis within the same category (Eg: within benign or malignant category) or to a different category (Eg: between benign and malignant category) especially for non-gynecology cases. All case pairs were also analyzed for a stepwise discrepancy in case of change of diagnosis to a different category as step 1, 2, 3, $4 \& 5$ to assess the severity. The cases with a change in diagnosis within the same category and step 1 discrepancy were grouped under no harm of severity of the error. The cases with step 2 and 3 discrepancies were grouped as near miss and step 4 and 5 was grouped as significant harm of severity of the error.

The results were entered in the Microsoft Excel sheet mentioning the parameters as; serial number, organ, cytology case number, sign-out original cytology diagnosis, date of cytology report, review cytology diagnosis, histopathology case number, sign-out original histopathology diagnosis, date of histopathology report, review histopathology diagnosis, time interval between cytology and histopathology diagnosis, concordant and discordant cases, dichotomous assessment of root cause analysis of discordant cases, sig- nificance/outcome of discrepancy or severity of error and error frequency. The effect of discrepancy or severity of error was classified as no harm, near miss and significant harm. The error frequency was calculated by three different methods ${ }^{2,3}$. The descriptive statistical analysis was done to establish the relationship between the cytology and histopathology reports.

\section{RESULTS}

A total of 639 case pairs were considered for the study of which gynecology cases (Pap test) were 447, and non-gynecology cases were 192 . The percentage of discordant cases was $4.92 \%$ and $13.02 \%$ in gynecology and non-gynecology cases respectively. The time interval between cytology and histopathology reports were 20.78 and 30.91 days in gynecology and nongynecology cases respectively. Among the discordant cases in both gynecology and non-gynecology case pairs, the interpretation error was more $(n=10$ and 15) compared to sampling error $(n=8$ and 9$)$ of which cytology interpretation error was more than histopathology interpretation error (Table 3). Table 3 also shows the severity of error in case pairs which were classified as no harm, near miss and significant harm. No harm was 13 and 15 cases in gynecology and non-gynecology case pairs respectively. The severity of error (significant harm) was more in non-gynecology cases $(n=6)$ compared to gynecology cases $(n=2)$. Organ-wise analysis of non-gynecology cases showed maximum discordant cases in the breast (28.0\%, $\mathrm{n}=7$ ) followed by the thyroid $(24.0 \%, \mathrm{n}=6)$ with the discordant concordant ratio of 1:5.85 and 1:4.83 respectively. The mean time interval in breast and thyroid case pairs were 28.1 and 23.1 days respectively. The change to same and different category following $\mathrm{CH}$ correlation in the breast was two and five cases respectively, and in thyroid, it was two and four respectively (Table 4). Of the seven case pairs of the breast, four had sampling error (core needle biopsy), and three had interpretation error (2 - cytology and 1 - histopathology). Of the six case pairs of the thyroid, five had interpretation error in cytology and one interpretation error in histopathology. For the majority of gynecology cases the time interval between cytology and histopathology reports was 11 to 20 days and for non-gynecology cases zero to ten days. The percentage of concordant and discordant cases approximately decreased and increased respectively with an increase in the time interval between cytology and histopathology reports. In gynecology case pairs, the concordance decreased from $94.90 \%$ to $60 \%$, and discordance increased from $5.09 \%$ to $40 \%$. 
Table 1: Shows stepwise classification of lesions in gynecology cases in cytology and corresponding terms in histopathology ${ }^{1}$

\begin{tabular}{lll}
\hline Steps & Cytology diagnosis & Histopathology diagnosis \\
1 & NILM & Benign \\
2 & ASC-US / AGC & No equivalent \\
3 & LSIL / AGC-FN & LSIL (CIN 1) / LGIL \\
4 & HSIL / In-situ glandular lesion & HSIL (CIN 2 \& 3) / HGIL \\
5 & Carcinoma & Invasive carcinoma \\
\hline
\end{tabular}

NILM: Negative for intraepithelial lesion and malignancy; ASC-US: Atypical squamouscells of undetermined significance; AGC: Atypical glandular cells; LSIL: Lowgrade squamous intraepithelial lesion; AGC-FN: Atypical glandular cellsfavourneoplasia; HSIL: High grade squamous intraepithelial lesion; CIN: Cervical intraepithelial neoplasia; LGIL: low grade glandular intraepitheliallesion; HGIL: High grade glandular intraepithelial lesion

Table 2: Shows stepwise classification of lesions in non-gynecology cases in cytology and corresponding terms in histopathology ${ }^{2}$

\begin{tabular}{lll}
\hline Steps & Cytology Diagnosis & Histopathology Diagnosis \\
1 & Normal cells & Normal tissue \\
2 & Inflammatory / Non-neoplastic & Inflammatory / Non-neoplastic \\
3 & Benign & Benign \\
4 & Atypical & Atypical (Infrequently used) \\
5 & Suspicious & Suspicious (Infrequently used) \\
6 & Malignant & Malignant \\
\hline
\end{tabular}

In non-gynecology case pairs, the concordance decreased from $91.93 \%$ to $83.3 \%$, and discordance increased from $8.06 \%$ to $16.6 \%$ (Table 5). Table 6shows error frequency in gynecology and non-gynecology case pairs calculated by different methods. Nongynecology cases had more percentage of error frequency (13.02 / 0.42 / 14.9) compared to gynecology cases $(4.92$ / 0.37 / 5.17).

\section{DISCUSSION}

CHC is a quality indicator of the Pathology laboratory. It is a method to detect the medical error of accuracy which is the difference between the cytology and histology diagnosis in original and review diagnosis and between two pathologists ${ }^{1,3,5,6}$. It compares the cytology and histopathology interpretation of the specimen from the same anatomic site and looks for concordance or discordance of the reports. It is a secondary process, i.e. post sign out process done for hospital patient usually for laboratory accreditation, tumor board, conference presentation, external review practices, internal assurance policy (as the clinical review of the set of cases, intradepartmental case discussion, review of misdiagnosis) and on physician request ${ }^{1,3}$.

It is a step-wise method which includes all activities that affect the quality of cytology and histology diagnosis, i.e., pre-examination phase involving patient identification, specimen procurement, transport and examination phase as specimen processing and interpretation by individual Pathologist and postexamination phase as release/review of reports. All the discordant case pairs are considered as errors ${ }^{1,6}$. Diagnostic errors result in wrong management and delay in a specific diagnosis ${ }^{2}$.

Anatomic pathology error reported is $1 \%$ to $43 \%$ of all anatomic pathology specimens. Mean anatomic pathology error frequency reported is $6.7 \%{ }^{3}$. CLIA ' 88 has mandated the use of $\mathrm{CHC}$ to improve anatomic pathology quality especially cytology diagnosis. It has started to correlate the Pap test with histologic specimens ${ }^{4,5}$. CAP has stated that $\mathrm{CHC}$ is a quality assurance activity in cytopathology labs ${ }^{4}$.CHC is a secondary viewing activity, error detection procedure, a prevention tool and is a lab patient safety armamentarium ${ }^{4}$.

Method of error detection is done in a dichotomous manner as follows: 


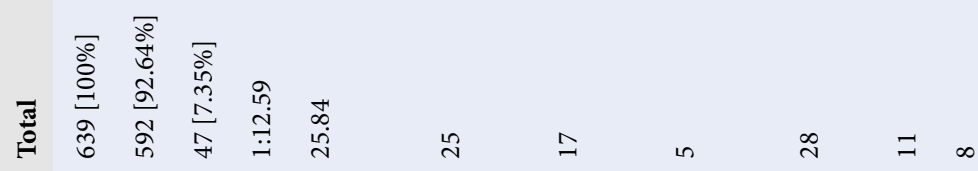

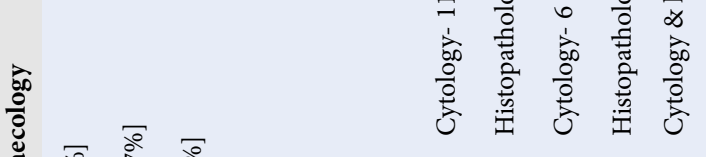

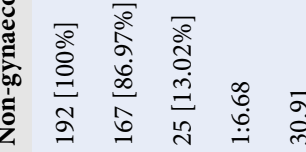
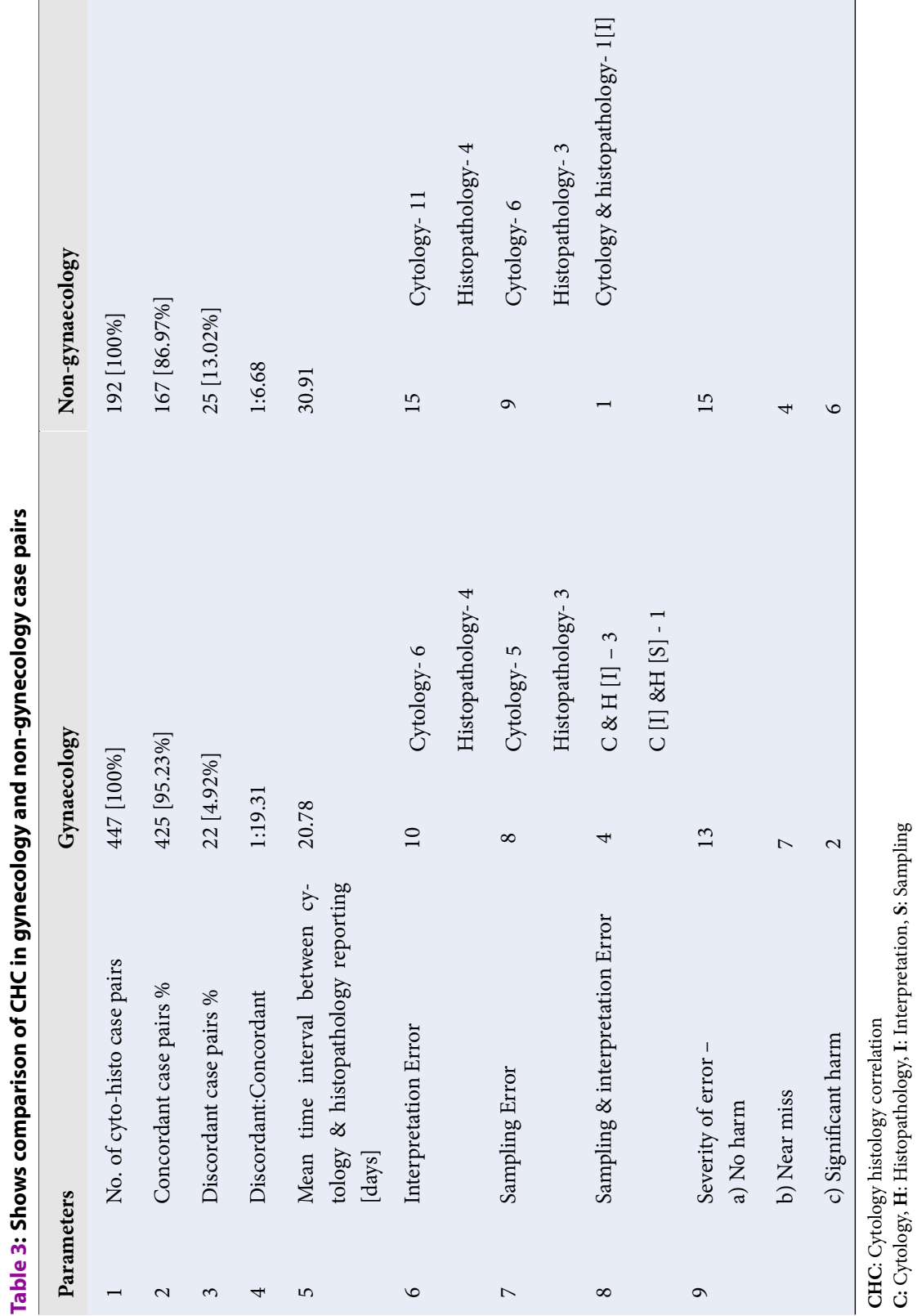

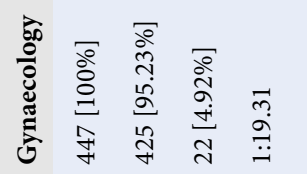
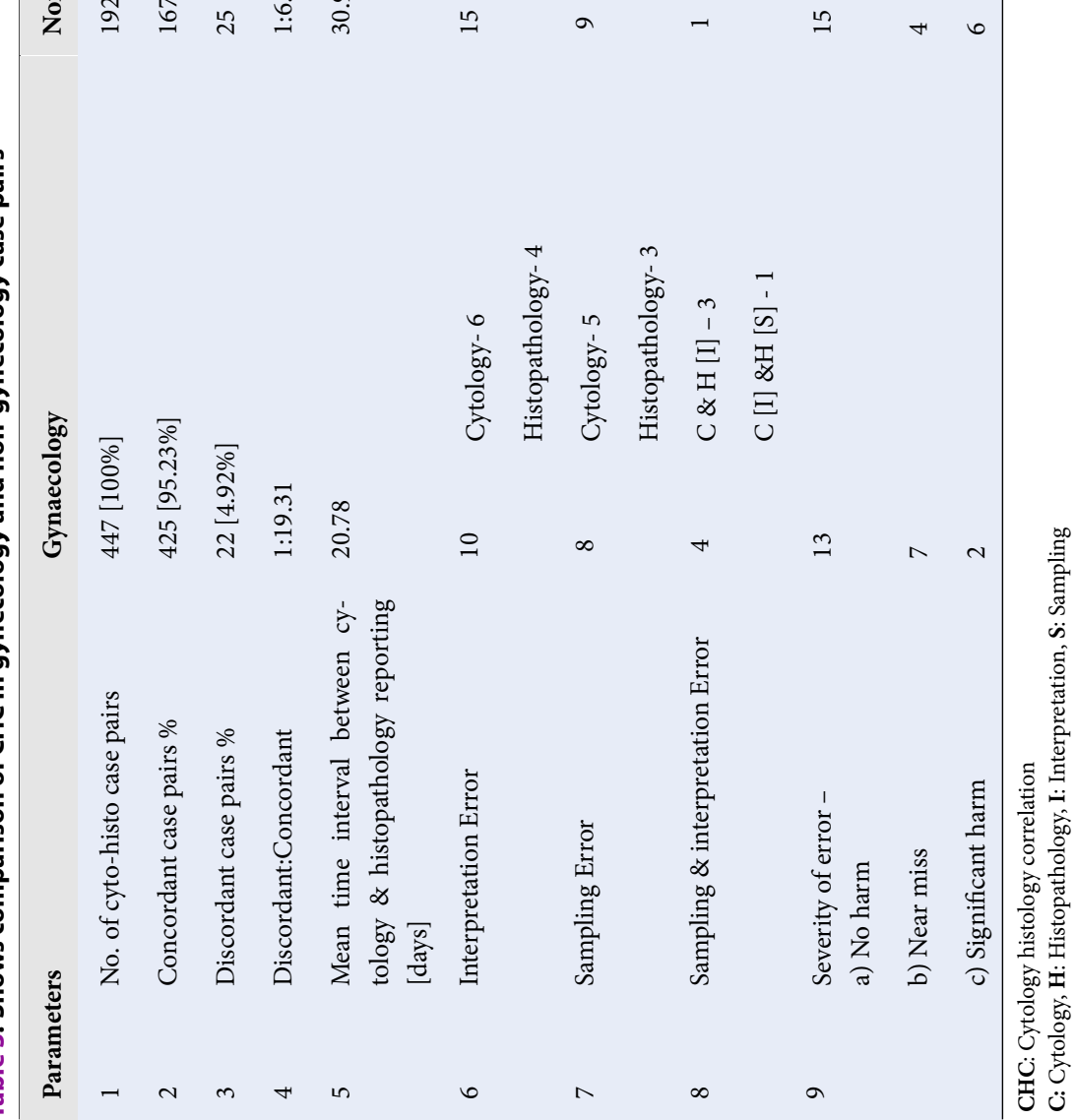


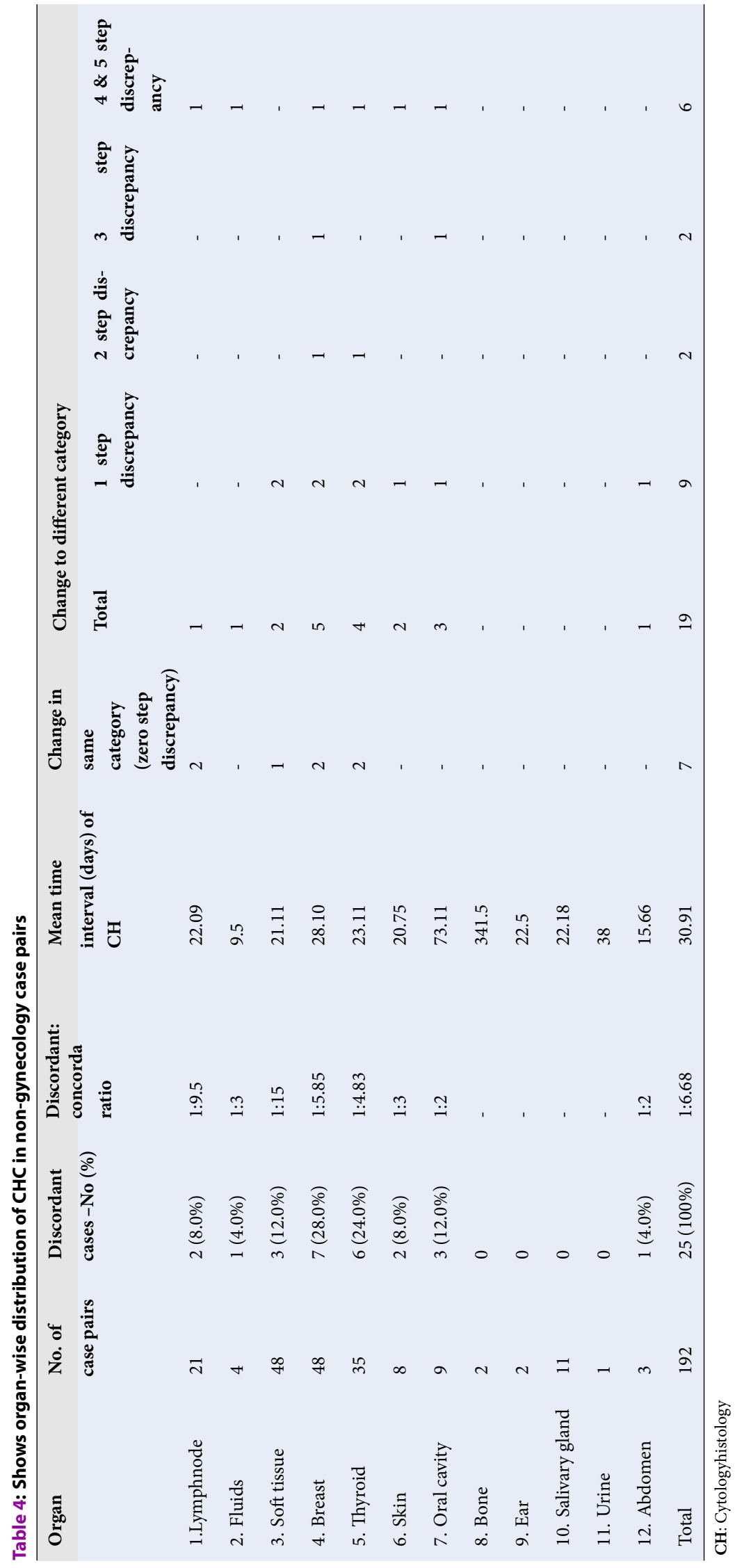




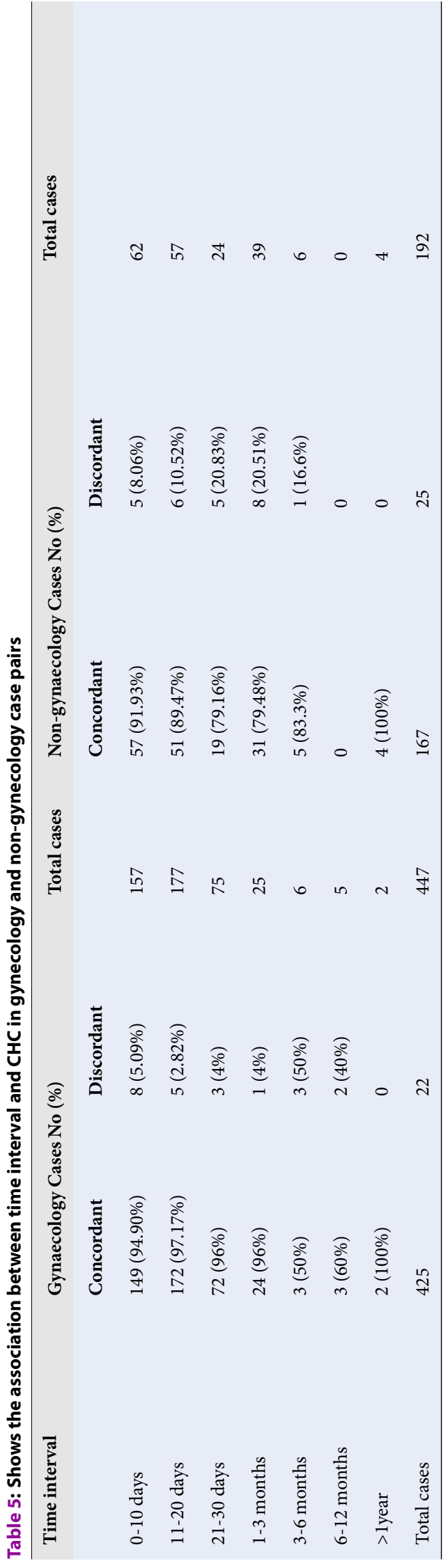


1. Active (looks errors in progress and detects highfrequency errors) and passive (detects errors after they happen)

2. Retrospective and prospective

3. Self-reporting and third-party reporting.

The CHC is retrospective, passive error detection through third-party reporting. CHC can be used as a prospective activity on histopathological specimens before the sign out of the report with antecedent cytology report as a form of quality improvement ${ }^{1,2}$. The present study is a retrospective study.

Several methods are followed for detection of discordance; secondary review method is common, and many times these are used for cancer diagnosis. The causes of the discordance can be classified in a dichotomous division (traditional binary method) as interpretative discordance (cognitive task), sampling/processing error (Clinician and technical skill discordance) and both. Each entity can be further classified as cytological, surgical, both and neither. Interpretation error is misclassified error (corrected diagnosis / error in diagnostic categorization). Interpretation error depends on inadequate diagnostic material, diagnostic material not present on the slide, material not properly interpreted, obscuring elements, Pathologists, and experience of the original and review pathologist examining the same slide. Sampling error includes no material on the slide due to non-procurement of material and/or lapses in proper processing of material before the diagnostic interpretation. Sampling error depends on the nature of the lesion, sampling device used, a method of processing the sample and expertise of the clinician. Hence, discordance occurs when pathologists interpret poor quality or non-representative biopsies. Therefore, structured interpretation should be used to decrease the error ${ }^{1-7}$. As per the CAP Q-probes study, most errors were secondary to sampling ${ }^{4}$. A study has reported that among the gynecologic case pairs, inadequate sampling in Pap tests is the primary root cause for error and improving this issue leads to the largest degree of improvement. Some studies have stated that liquid-based cytology technique has improved the detection of the pre-neoplastic lesion and hence decreased the error ${ }^{5}$. In the present study, interpretation error was more than sampling error in both gynecology and non-gynecology cases. The error was more in cytology interpretation than biopsy interpretation.

The determination of root cause for discordance is difficult and limited which also depends on the reproducible or non-reproducible diagnosis. One has to look for all the steps in the testing process, determine where the error has occurred, and rectification of error which can be pre-examination or examination process will help in quality improvement. The use of non-standardized diagnostic criteria in assessing the adequacy of FNA specimens considering the cellularity, background, and preservation of cells immediately after aspiration correlating with radiology findings will to some extent reduces the error of sampling. The institutional error is due to variable clinical and laboratory practices and partly due to biases ${ }^{2}$.

The rate of discordance in gynecologic and nongynecologic case pairs reported were 1.79 to $9.42 \%$ and 4.87 to $11.8 \%$ respectively in one study ${ }^{2}$. The $\mathrm{CHC}$ discordance many times depends on the institution as some institutions have high error frequency probably because of the difference in standards of data collection and processes. Labs with increased $\mathrm{CHC}$ discordant frequency probably may have a better capacity of detecting error than being of poorer quality. In one study the interpretation error was $5.0-50.7 \%$ and rest was sampling error ${ }^{2,4}$. Clary stated that among non-gynecologic Cytologyhistology $(\mathrm{CH})$ case pairs, $2.3 \%$ error were in cytology samples and $0.44 \%$ in surgical samples ${ }^{1,2,4}$. The discrepancy in the cytology specimen of original diagnosis results in the clinical effect. One study has reported the mean and median lab discrepancy frequencies were $6.7 \%$ and $5.1 \%$ respectively. Mean multi-institutional discrepancy frequency was $6.7 \%$. McBroom \& Ramsay reported $9.0 \%$ of the discrepancy $^{3}$. The multinational consortium has reported that among the discordance of $\mathrm{CH}$ cases, $5 \%$ to $42 \%$ constituted diagnostic (interpretation) errors and the rest constituted sampling error ${ }^{6}$. Tables 7 and 8 show discordant and concordant cases in different institutions compared to the present study indicating discordance in non-gynecology cases were more in the present study compared to other studies.

$\mathrm{CHC}$ discordance also depends on the anatomic site of the sample/specimen ${ }^{1}$. The anatomic pathologic errors reported is 1 to $43 \%$ among all specimens ${ }^{2}$. The frequency of error in reporting lung mass is approximately $15 \%$ and it is mainly due to inter-observer variation $^{8}$. CH discrepancy for lungs reported was $6 \%$ to $17 \%$ and the majority of the discordance was due to cytology than histopathology sampling/ interpretation. Regarding urine analysis, the discordant rate was $40.9 \%$, interpretation error was $35 \%$, sampling error was $63 \%$, and minimal/mild harm was $48.6 \%^{1}$. Some organs as FGT and breast have higher discrepancy resulting in clinical harm compared to other organs. Clary stated that most errors that are associated 


\begin{tabular}{llll} 
Table 6: Shows error frequency in gynecology \& non-gynecology case pairs calculated by different methods \\
\hline Error frequency & Gynaecology (\%) & Non-gynaecology (\%) & Total (\%) \\
Method 1 & 4.92 & 13.02 & 7.3 \\
Method 2 & 0.37 & 0.42 & 0.79 \\
Method 3 & 5.17 & 14.9 & 7.9
\end{tabular}

Method 1: Number of correlation errors of CH case pairs / Total Number of discordant \& non- discordant CH case pairs Method 2: Number of correlation errors of $\mathrm{CH}$ case pairs / Total number of cytology cases (Cytology work load)

Method 3: Number of correlation errors of $\mathrm{CH}$ case pairs / Total number of correlating cases

Table 7: Shows error frequency of $\mathrm{CH}$ discordant case pairs in different studies compared to present study

\begin{tabular}{lllllll}
\hline Studies & \multicolumn{2}{l}{ Gynecology cases } & \multicolumn{3}{l}{ Non-Gynecology cases } \\
& I & II & III & I & II & III \\
Raab SS et al 2005 $^{2}$ & 4 & 0.30 & 4.00 & 10.8 & 3.10 & 10.8 \\
A institute $^{2}$ & 8.6 & 0.63 & 9.42 & 11.1 & 1.42 & 11.2 \\
B institute $^{2}$ & 1.7 & 0.14 & 1.79 & 5.4 & 1.18 & 5.75 \\
C institute $^{2}$ & 4.7 & 0.36 & 4.72 & 10.5 & 5.45 & 11.8 \\
D institute $^{2}$ & 2.6 & 0.17 & 2.73 & 4.6 & 1.69 & 4.87 \\
Present study 2018 $^{2}$ & 4.76 & 0.37 & 5.17 & 13.02 & 0.42 & 14.9 \\
\hline
\end{tabular}

I: Denominator is total Number of discordant \&non- discordant CH case pairs

II: Denominator is total number ofcytology cases (Cytology work load)

III: Denominator istotal number of correlating cases

Table 8: Shows $\mathrm{CH}$ correlation studies done in various institutes $[\mathrm{A}, \mathrm{B}, \mathrm{C}, \mathrm{D}]^{2}$ compared to present study $[\mathrm{P}]$

\begin{tabular}{llllllllllll}
\hline CH correlation & \multicolumn{3}{c}{ Gynecology case pairs } & \multicolumn{1}{c}{ Non-gynecology case pairs } \\
& A & B & C & D & P & A & B & C & D & \\
Discordant cases & 139 & 103 & 430 & 18 & 22 & 74 & 100 & 477 & 23 & 25 \\
Concordant cases & 1476 & 5748 & 9119 & 660 & 425 & 662 & 1740 & 4033 & 472 & 167 \\
Discordant:concordar & $1: 10.6$ & $1: 55.8$ & $1: 21.2$ & $1: 36.6$ & $1: 19 .:$ & $1: 8.9$ & $1: 17.4$ & $1: 8.4$ & $1: 20.5$ & $1: 6.6$ \\
\hline
\end{tabular}

A: A institute; B: B institute; C: C institute; D: D institute; P: Present institute

with harm were of lungs and breast cytology specimens. Tronel and Sabella stated that diagnostic errors in breast cytology were associated with harm. The other organs with similar reports were prostatic needle biopsy, Pap test, and melanocytic skin lesions ${ }^{3}$. In the present study, breast and thyroid had maximum discordance and was associated with no harm Table 4. Table 9 shows the organ-wise comparison of discordant cases in the present study with other studies.

The discordance also depends on the time interval between the cytology and histology report as longer the interval lesser the chances of detection of discordance and the percentage of the discrepancy. Sometimes one cytology report has one or two histopathology report ${ }^{1}$. In the Q-tracts gynecology histologic correlation study, it is stated that the Pap test has to be performed within three months before the biopsy or at the time of biopsy as this time interval reduces the errors compared to six months window period. However, the maximum time interval in non-gynecologic case pairs to do CHC is not defined ${ }^{4}$. The present study also shows an increase in discordant cases with time. However, it is not that significant Table 5.

A study has reported that $48 \%$ discordance was due to change within the same category of interpretation and $21 \%$ was due to change across categories indicating that change in the same category of diagnosis was the most common discrepancy detected. Change in category occurred more frequently with female genital tract (FGT), male genital tract (MGT) and lymph node. The other findings of the cause of discordance can be changed in margin status, change in patient information and topographic error which can result in harm to the patient ${ }^{3}$. In the present study, among 


\begin{tabular}{|c|c|c|c|c|}
\hline Organ & $\begin{array}{l}\text { Raab SS et al } \\
2005^{3}\end{array}$ & $\begin{array}{l}\text { Raab SS et al } 2005^{2} \text { [A } \\
\text { Institute] }\end{array}$ & $\begin{array}{l}\text { Raab SS et al } 2005^{2} \text { [C } \\
\text { Institute] }\end{array}$ & $\begin{array}{l}\text { Present study } \\
2018\end{array}$ \\
\hline Breast & $8.3 \%$ & $15 \%$ & $13 \%$ & $28.20 \%$ \\
\hline Soft tissue & $7.5 \%$ & $0 \%$ & $4 \%$ & $12.0 \%$ \\
\hline Lymph node & $5.9 \%$ & $5 \%$ & $16 \%$ & $8.0 \%$ \\
\hline Urine & $7.2 \%$ & $11 \%$ & $25 \%$ & $0 \%$ \\
\hline Oral cavity & $5 \%$ & - & - & $12.0 \%$ \\
\hline Thyroid & $8 \%$ & $0 \%$ & $11 \%$ & $24.0 \%$ \\
\hline Bone & $2 \%$ & $0 \%$ & $4 \%$ & $0 \%$ \\
\hline Salivary gland & $3.5 \%$ & - & - & $0 \%$ \\
\hline $\begin{array}{l}\text { Total for non- } \\
\text { gynecology cases }\end{array}$ & $6.7 \%$ & - & - & $13.02 \%$ \\
\hline
\end{tabular}

Table 10: Shows grade of severity for CH discordant gynecology \& non-gynecology cases in different studies compared to present study

\begin{tabular}{|c|c|c|c|c|c|c|}
\hline \multirow[t]{2}{*}{ Studies } & \multicolumn{3}{|c|}{ Gynecology case pairs } & \multicolumn{3}{|c|}{ Non-gynecology case pairs } \\
\hline & No harm & $\begin{array}{l}\text { Near } \\
\text { miss }\end{array}$ & Significant & No harm & Near miss & Significant \\
\hline Raab SS et al $2005^{2}$ & $46 \%$ & $8 \%$ & $45 \%$ & $55 \%$ & $5 \%$ & $39 \%$ \\
\hline Raab SS et al $2005^{3}$ & - & - & - & $69.9 \%$ & $4.8 \%$ & $25.3 \%$ \\
\hline Present study 2018 & $59.09 \%$ & $31.81 \%$ & $9.09 \%$ & $20 \%$ & $32 \%$ & $54.54 \%$ \\
\hline
\end{tabular}

CH: Cytology histology

non-gynecology cases, change across category was more common $(\mathrm{n}=19)$ than within the same category $(n=7)$ and it was more in breast and thyroid cases Table 4 .

The discrepancy with the change in the category can be analyzed as stepwise diagnostic discordance and correlated with clinical significance and degree of harm to the patients. Higher the step difference, greater will be the probability of severity of clinical outcome as benign and atypia (one step) versus benign and malignant (three steps) ${ }^{1,2}$. Two-step discrepancies have a greater probability of clinical significance than one step variability. Interobserver variability studies with one step discrepancy are much more common $^{3}$. In CAP Q-probe study, one step or higher and two steps or higher discordance are reported in $30.8 \%$ and $3.6 \%$ respectively in $\mathrm{CH}$ case pairs excluding atypical Pap test cases and histologic interpretation failures. Agency for Health Care Research and Quality Laboratory Consortium has reported that the discordance in two-step or greater correlation was of $4.0 \%$ and $45 \%$ of this discordance is due to delay in diagnosis or due to unnecessary procedures ${ }^{5}$. The severity of clinical significance can be categorized as; No harm regardless of an erroneous diagnosis, near miss where it is intervened before harm could have occurred or not acted on an erroneous diagnosis and significant harm which can be minimal, moderate and severe ${ }^{1-3} .23 \%$ interpretation error among non-gynecology case pairs had a major effect on patient care ${ }^{2,4}$. It has been reported that up to $50 \%$ of the errors result in mild to moderate harm ${ }^{7}$. A study has reported that $5.3 \%$ of discordance has resulted in moderate to marked effect on patient care. Raab reported an error frequency of $8.9 \%$ and severe clinical significance events in $7.0 \%$ of all errors ${ }^{3}$. The comparison of the severity of the clinical significance of various studies with the present study is shown in Table 10. The error frequency reported in oncologic Pathology ranges from $1 \%$ to $15 \%{ }^{7}$. In the present study, the error frequency is calculated using a different method as shown in Table 6.

The other recent method of analyzing the discordance is the continuous assessment of factors i.e. No-Blame 
box method of root cause analysis which considers two important factors that determines sampling error or interpretation error and act as quality indicators to detect system problem than only active human interpretation failures i.e. specimen quantity (amount of tumour tissue) and the quality of specimen for both cytology and histopathology ${ }^{1}$. The compatibility of specimen interpretation among Pathologists ranged from $13 \%$ to $20 \%$, and the majority of discordance were due to un-interpretable samples. Use of NoBlame Box showed significant improvement with respect to the agreement among Pathologists in finding the cause of discordance and the method of root cause analysis of discordance cases ${ }^{6}$. The other method of root cause analysis is by system assessment, i.e. assessment of system components of failure which has many methods ${ }^{1}$.

$\mathrm{CHC}$ studies have certain disadvantages. Not all the $\mathrm{CH}$ cases received by the laboratory are considered for CHC. Hence, one cannot accurately assess the frequency of error. CHC detects more cytology error than histopathology error. There is no specific standard method to conduct CHC, not governmentally regulated, no standard results are obtained, poorly characterized to date, limitation of single institutional reporting and the same is not outlined in CLIA'88 regulations. It also depends on the laboratory facility with Laboratory information system (LIS) to track $\mathrm{CHC}$ pairs. The disparity in the collection of clinical and pathological variables at different centers may affect data collection and in turn institutional performance. Hence, the data obtained is difficult and impossible to compare between laboratories. The future of $\mathrm{CHC}$ lies on the use of national/global standardized methods, the robust process of RCA of discordant cases and redesign the testing process when required for quality improvement. Because of the lack of correlation guidelines in CHC, it cannot be used as "Best Practice" and data for self-improvement. The other limitations are lack of understanding when an error has occurred, fear of disclosure and bias in error detection ${ }^{1-5,8}$. It is difficult to measure the true frequency of error because of the different detection methods used and the definition of error ${ }^{2,3}$. Many times there is a lack of subspecialty expertise ${ }^{3}$. Pathologist exhibits very poor agreement in concluding the root cause of $\mathrm{CHC}$ discordance. Therefore, it is difficult to design the guidelines to reduce the error frequency. There is a lot of variability in institutional reportability and consensus of the root cause of the discrepancy. Many times the discrepancy is due to system failure than the incompetence of Pathologist or clinician incompetence ${ }^{6,8}$.
The advantages of $\mathrm{CHC}$ studies are, the data can be used to assess the performance of the laboratory as sensitivity and specificity ${ }^{1}$. The advantages of $\mathrm{CHC}$ studies are an analysis of frequency and cause of discordance which has clinical impact. Error reduction strategies can be done by qualitative and quantitative method. The scope of establishing accurate method for original and review diagnosis and knowledge of clinical outcome in reporting pathologist helps in quality patient care ${ }^{2-4}$. Error prevention programmes help with good patient care ${ }^{3}$. Pathology reporting, patient safety and assessment of patient outcome are triads of $\mathrm{CHC}$. CHC is ideal for the pathologist to self-assess the errors ${ }^{3}$. Monitoring of $\mathrm{CHC}$ for a long time is associated with improvement in performance of CHC. It improves detection of preneoplastic lesions of the institute. The Institute of Medicine Report has agreed for the establishment of a national error reporting system as an initial step to improve patient safety globally ${ }^{5}$.

\section{CONCLUSIONS}

In the present study, the percentage of error in gynecologic cases is within the range of published data. However, those of non-gynecology cases are slightly higher, maximum in breast cases followed by the thyroid. Breast cases had more of biopsy sampling error than interpretation error. All thyroid cases had interpretation error; five of cytology and one histopathology. Regular professional training and improvement of sampling techniques especially the guided FNAC can reduce the errors. Hence, both clinicians and Pathologists are equally responsible for the reduction of errors and improve patient care. Understanding the root cause and addressing it is the most important activity.

\section{ABBREVIATIONS}

CHC: Cytology Histology correlation

CLIA: Clinical laboratory Improvement Amendments

QC: Quality Control

RCA: Root cause analysis

FNAC: Fine needle aspiration cytology

Pap Test: Papanicolaou test

CH: Cytology Histology

CAP: College of American Pathologists

FNA: Fine needle aspiration

FGT: Female genital tract

MGT: Male genital tract

\section{COMPETING INTERESTS}

The authors report no conflicts of interest in this work. 


\section{AUTHORS' CONTRIBUTIONS}

Kalyani Raju: Concept, data collection, writing the script, editing; Supreetha MS: Data collection; Varshashree R: data collection.

\section{REFERENCES}

1. Raab SS, Grzybicki DM. Cytologic-histologic correlation. Cancer Cytopathol. 2011;119(5):293-309. 21732549. Available from: 10.1002/cncy.20165.

2. Raab SS, Grzybicki DM, Janosky JE, Zarbo RJ, Meier FA, Jensen $C$, et al. Clinical impact and frequency of anatomic pathology errors in cancer diagnoses. Cancer. 2005;104(10):2205-13. 16216029. Available from: $10.1002 / \mathrm{cncr} .21431$.

3. Raab SS, Nakhleh RE, Ruby SG. Patient safety in anatomic pathology: measuring discrepancy frequencies and causes. Arch Pathol Lab Med. 2005;129(4):459-66. 15794667.

4. Vrbin CM, Grzybicki DM, Zaleski MS, Raab SS. Variability in cytologic-histologic correlation practices and implications for patient safety. Arch Pathol Lab Med. 2005;129(7):893-8. 15974812.

5. Raab SS, Jones BA, Souers R, Tworek JA. The effect of continuous monitoring of cytologic-histologic correlation data on cervical cancer screening performance. Arch Pathol Lab Med. 2008;132(1):16-22. 18181668

6. Raab SS, Stone CH, Wojcik EM, Geisinger KR, Dahmoush L, Garcia FU, et al. Use of a new method in reaching consensus on the cause of cytologic-histologic correlation discrepancy. Am J Clin Pathol. 2006;126(6):836-42. 17074685. Available from: 10.1309/1790JN2YWCG833VU.

7. Raab SS, Grzybicki DM. Quality in cancer diagnosis. CA Cancer J Clin. 2010;60(3):139-65. 20444999. Available from: 10.3322/ caac. 20068.

8. Raab SS, Meier FA, Zarbo RJ, Jensen DC, Geisinger KR, Booth CN. The "Big Dog" Effect: Variability Assessing the Causes of Error in Diagnoses of Patients with Lung Cancer. J Clin Oncol. 2006;24(18):2808-14. 16782918. Available from: 10.1200/JCO. 2005.04.3661. 